\title{
MÉXICO, CANADÁ Y LA SEGURIDAD EN AMÉRICA DEL NORTE Athanasios Hristoulas*
}

RESUMEN: El objetivo de este artículo es demostrar las diferencias tan marcadas que existen entre el acercamiento de Canadá y el de México en torno a la seguridad de Norteamérica; argumenta que México enfrenta una batalla cuesta arriba en el esfuerzo de ser un buen aliado en la lucha contra el terrorismo.

PALABRAS ClAVE: seguridad, terrorismo, agencias de seguridad, cooperación, fronteras.
ABSTRACT: This article intends to show the pronounced differences between Canada and Mexico's approaches regarding North American security. It further states that Mexico faces an uphill battle in its efforts of supporting the fight against terrorism.

KEYWORDS: security, terrorism, security agencies, cooperation, borders.
RECEPCIÓN: 25 de noviembre de 2008. ACEPATACIÓN: 21 de enero de 2009.
* Coordinador del Diplomado en Seguridad Nacional. Departamento de Estudios Internacionales, ITAM. 


\section{MÉXICO, CANADÁ Y LA SEGURIDAD EN AMÉRICA DEL NORTE}

Los ataques terroristas representan un punto crítico en la política exterior mexicana. Rompiendo con su tradición de no-intervención, México tomó medidas para demostrar políticamente su solidaridad con los Estados Unidos. Uno de ellos, por ejemplo, fue cuando en octubre de 2001 el Presidente Fox declaró que México "considera la lucha contra el terrorismo como parte del compromiso que tiene México con Canadá y Estados Unidos para construir, dentro del marco del Tratado de Libre Comercio de América del Norte, un espacio compartido del desarrollo del bienestar y la seguridad integral." Posteriormente, en ese mismo año, Jorge Castañeda, en este entonces Secretario de Relaciones Exteriores, dijo que
"México favorecería un acercamiento continental a las cuestiones de seguridad fronteriza, extendiendo una sociedad norteamericana que ya opera a nivel comercial." En ese mismo discurso, Castañeda señaló que el gobierno mexicano prefería llevar un perímetro de seguridad "tan lejos como sea posible, pero ello depende de los canadienses y de los americanos."

¿Por qué este cambio? La respuesta está en que el gobierno mexicano vio en el contexto de la seguridad continental y fronteriza una gama de oportunidades en las áreas de comercio, seguridad, migración e incluso desarrollo social. Lo que le preocupaba a México era que la seguridad intensificada en la frontera lastimara el comercio entre los Estados Unidos 
y México, y por este motivo debía contribuir en la formación del perímetro de seguridad. Los intereses de México fueron más allá del intercambio económico; la estrategia ha sido tal que encadene los problemas o el intento de intercambiar seguridad por otro tipo de beneficios. El tema más importante de este intento de encadenar está directamente relacionado con la expansión del TLCAN para incluir otras cuestiones no relacionadas con el comercio.

Cuando la administración de Salinas firmó el TLCAN a principios de los noventa, su administración argumentó que un acuerdo trilateral resultaría en un mejoramiento para la calidad de vida de los mexicanos. Dos sexenios más tarde, la administración de Fox se vio fuertemente presionada por cumplir las promesas hechas por Salinas. En los meses siguientes del los atentados terroristas, Fox argumentó en repetidas ocasiones que mientras México fuera un lugar donde el $40 \%$ de la población ganara menos de dos dólares por día, las fronteras de EU no estarían seguras. En consecuencia la solución es un acuerdo migratorio donde EU legalmente absorba a un número importante de migrantes mexicanos, o un programa de cohesión social en Norteamérica similar al que existe en la Unión Europea o, preferiblemente, ambos. Impulsando esta idea de encadenamiento aún más, los toma- dores de decisión mexicanos llegaron a tales extremos como argumentar que en el interés de la seguridad nacional norteamericana, EU debía legalizar a 4 millones de trabajadores indocumentados mexicanos porque resultaba mejor conocer quiénes eran realmente, dado el contexto de defensa nacional. En consecuencia, la migración es la clave para entender el fuerte apoyo de México en materia de cooperación con la seguridad continental y fronteriza.

No obstante, México no puede mantenerse al nivel de Canadá y Estados Unidos ni políticamente ni operacionalmente con los cambios que ocurren en Norteamérica. Existe, en otras palabras, una fuerte desvinculación entre la retórica mexicana y su acción. Primero, tanto México como Canadá firmaron con Estados Unidos los acuerdos de "frontera inteligente" en 2001 y 2002, diseñados con el fin de hacer la región fronteriza más segura y más eficiente. Aunque el acuerdo entre Estados Unidos y México es más modesto en sus objetivos, ha habido una gran dificultad en concretizar varias partes del acuerdo. Por ejemplo, mientras que en el caso de Canadá con Estados Unidos, ya se han llevado a cabo prácticamente los 32 puntos del acuerdo, o están en proceso de hacerlo, la contraparte mexicana se ha estancado. De los 22 puntos en el acuerdo entre Méxi- 
NOTAS

co y Estados Unidos, no ha habido progreso alguno en siete áreas críticas. Esto incluye vías ferroviarias seguras, combate al fraude, interdicción del contrabando, intercambio electrónico de información, examinar a nacionales de países en vías de desarrollo y consultas a la política de visado.

Cuatro factores adicionales interrelacionados se combinan para concluir que México se enfrenta a una batalla cuesta arriba y que posiblemente pueda perder en el esfuerzo de convertirse en un socio confiable en la guerra contra el terrorismo dentro del contexto norteamericano. El primero es un sistema politico fragmentado; el segundo corresponde a la corrupción institucional; el tercero es la intensa y contraproducente compe-

142 tencia entre las diferentes agencias de seguridad; y el cuarto es el abuso político del nacionalismo y la soberanía por parte de los funcionarios $y$ politicos mexicanos para beneficio de su trayectoria.

Analizando la fragmentación política, primero, los seis años de la administración del Presidente Fox se caracterizaron por una intensa lucha política entre las diferentes secretarías; esto, aunado al hecho de que el Congreso estaba profundamente dividido, llevó a una situación en la cual los líderes del país fueron incapaces de tomar decisiones rele- vantes sobre el tema. Para fines de este ensayo, no sería inexacto argumentar que dicha fragmentación ha conducido a una situación donde el Presidente Fox básicamente salió del paso durante su período. Un ejemplo de ello es el caso de la estrategia para la Seguridad Nacional de México, seguida tras las elecciones presidenciales del 2000. Cuando el Presidente Fox tomó posesión de su cargo, intentó formalizar la agenda en cuestiones de seguridad nacional creando el cargo de Consejero Presidencial de Seguridad Nacional. Este acto significó un intento por desarrollar una doctrina coherente de seguridad nacional que reorganizara en forma racional a las diferentes agencias de inteligencia, que incluyen (hasta el momento) al Centro de Investigación y Seguridad Nacional (CISEN), Inteligencia Naval, Inteligencia del Ejército, Procuraduría General de la República (PGR), Policía Federal Preventiva (PFP) y, finalmente, la Agencia Federal de Investigación (AFI).

La política del presidente fracasó porque el asesor para la Seguridad Nacional, Adolfo Aguilar Zinser, fue extremadamente impopular con las agencias arriba mencionadas. Sin duda, el acceso de Aguilar Zinser a la comunidad de inteligencia fue intencionalmente limitada no sólo por el CISEN (que no lo quería en ese cargo porque lo veía como una amenaza directa) sino 
por otras agencias de inteligencia. Al final, el Consejero para la Seguridad Nacional dejó su cargo por un puesto diplomático ante la ONU en Nueva York; de manera más significativa, el presidente decidió que no era necesario un reemplazo. En una decisión irónica, sin embargo comunicativa, Fox resolvió autonombrase su propio consejero de seguridad. De esta forma, la lucha entre las agencias mencionadas así como su desinterés por el tema, condenaron al fracaso una reestructuración real de la doctrina de seguridad nacional y servicios de inteligencia.

Las diferentes agencias lograron remover a Zinser del cargo, no obstante el problema de fondo se mantuvo. $\mathrm{Y}$ es que existe un falta significativa de un mandato claro, así como actividades que se sobreponen y compiten en las diferentes agencias de inteligencia. La estructura no coordinada de inteligencia debilita la toma de decisiones; la información recolectada entre las diversas agencias es celosamente guardada y no existe un mecanismo centralizador que concentre, consolide y sintetice la inteligencia. Como es posible notar, la competencia entre las agencias es feroz. Aún así, la Secretaría de Marina ha tomado una actitud más abierta hacia la cooperación con los Estados Unidos en cuestiones de seguridad. Por otro lado, la Secretaría de Defen- sa Nacional ha seguido una política más tradicional e aislacionista. Esto ha llevado a repetidas confrontaciones entre ambas secretarías. Otros ejemplos adicionales incluyen una disputa pública entre Jorge Castañeda como Secretario de Relaciones Exteriores y Adolfo Aguilar Zinser como embajador de México ante las Naciones Unidas. La confrontación se centró en la posición de México vis-à-vis la guerra contra Irak. Mientras Castañeda estaba dispuesto a apoyar la idea de secundar a Estados Unidos, Zinser se opuso de manera inflexible y criticó abiertamente no sólo a los Estados Unidos sino al Secretario de Relaciones Exteriores. El drama fue visto por televisión nacional e incluso requirió por parte del Departamento de Estado de los Estados Unidos una petición aclaratoria sobre quién estaba a cargo de la decisión. Lo que resultó abundantemente claro para todos los observadores fue que Fox estaba políticamente paralizado por la disputa entre sus dos subalternos. Curiosamente, Fox fue acusado de dirigir un gabinete tipo “Montessori”. La percepción generalizada era que cada secretaría operaba de acuerdo con lo que cada secretario de estado quisiera, contrario a un criterio unificado.

La corrupción ocupa el segundo lugar en cuanto a los problemas, ya que ésta es particularmente feroz en 
NOTAS

el Instituto Nacional de Migración (la agencia responsable del control migratorio). Ejemplos de esta corrupción desenfrenada son, por mencionar alguno, el Aeropuerto Internacional de la Ciudad de México donde las personas pueden entrar ilegalmente al país sobornando a agentes de migración. De igual importancia es la condición de anarquía en la frontera sur del país, donde indocumentados de todo tipo, que intentan cruzar la frontera con Guatemala, se enfrentan al cruce de un río desprotegido y poco profundo. La naturaleza de esta frontera, que carece de control, ha fomentado la creación de "empresarios" profesionales (y por supuesto ilegales) en el cruce del río. Estas personas cobran 10 pesos (aproximadamente un dólar) por el paso y desde luego, lo pueden hacer porque no hay agentes de migración de ningún lado de la frontera.

La extorsión comienza una vez que los migrantes han entrado en territorio mexicano. En este punto deben de sobornar no sólo a criminales comunes sino a autoridades de migración, policías locales, estatales y militares para poder permanecer en el país (la mayoría de los cuales utilizan a México como un punto de tránsito hacia los Estados Unidos). Esta corrupción, ampliamente difundida, envía una señal incorrecta a Washington-incluso a Ottawa-respec- to a la capacidad de México de lidiar con el potencial problema terrorista. De manera irónica, la opinión pública mexicana y las autoridades critican continuamente el trato de los migrantes mexicanos por parte de los oficiales estadounidenses a lo largo de la frontera norte, mientras que permanecen completamente ajenos y sin pensar en la tragedia humana que ocurre en la frontera entre México y Guatemala.

La competencia entre agencias policíacas funge como un tercer impedimento. Como se mencionó anteriormente, la disputa entre la Secretaría de la Defensa Nacional y la Secretaría de Marina es un obstáculo pero no el único. Existe, de manera extraña, un debate inter-institucional entre las diversas agencias de inteligencia, policías y tribunales sobre la "posesión" de la agenda terrorista. Incapaces de cooperar entre ellas, la "guerra" contra el terrorismo en México ha degenerado en una competencia entre agencias sobre la jurisdicción de diferentes partes de la agenda. Esto influye negativamente sobre el siguiente punto.

$\mathrm{Si}$ a los funcionarios mexicanos les resulta complicado lidiar unos con otros, la situación con los oficiales estadounidenses es peor. Pocos, si es que hay, mecanismos para la cooperación y comunicación existen entre 
las autoridades mexicanas y estadounidenses a nivel local. Tanto los funcionarios mexicanos como estadounidenses tiene problemas de confianza muy arraigados. Poca comunicación e intercambio de información existe entre ambos lados, llevando frecuentemente a confrontaciones potencialmente peligrosas. Los oficiales de EU se encuentran habitualmente en situaciones frustrantes, incapaces de lidiar con la ineficiencia que generalmente caracteriza a los oficiales mexicanos. Por su parte, las autoridades mexicanas son sumamente sensibles al unilateralismo de EU y carecen de la pericia y experiencia para fomentar los mecanismos de cooperación que existen a lo largo de la frontera entre Canadá y EU. El efecto final es que no hay confianza en la seguridad a lo largo de la frontera entre EU y México, y la cooperación bi-nacional se concentra en reducir fricciones y malentendidos entre los cruces fronterizos en vez de operaciones coordinadas.

Sin duda, los problemas entre la frontera de Estados Unidos y México no comenzaron con el once de septiembre. Uno de los conflictos más persistentes en la relación ha sido el narcotráfico. Desde el inicio de la "guerra contra el narcotráfico" en los años setenta, México y los Estados Unidos han tenido una percepción distinta de la naturaleza de la amenaza y de cómo lidiar de la mejor manera con ella. Para los Estados Unidos el problema es esencialmente una cuestión de eliminar el consumo de sustancias ilícitas domésticamente, mientras que para México, el narcotráfico ha sido visto históricamente dentro de la óptica de las posibles ramificaciones legales y políticas (en otras palabras, la capacidad del narcotráfico para debilitar las instituciones estatales). Esta diferencia de perspectivas de la naturaleza del problema ha creado confusión con respecto al intento de coordinar estrategias bilaterales de combate al narcotráfico.

El obstáculo final es la utilización del nacionalismo y la soberanía por parte de los actores políticos mexicanos con el fin de seguir agendas personales. Ejemplos de esto son notorios, pero este ensayo sólo mencionará uno. En el 2003, una banda de jóvenes del lado mexicano de la frontera robaban con regularidad un tren en las afueras de Nogales con destino a Estados Unidos. Después de múltiples robos, las autoridades estadounidenses solicitaron la asistencia de autoridades locales y estatales mexicanas que estuvieron más que entusiasmados en participar en la operación conjunta. Sin embargo, después de unas semanas, miembros del Congreso Federal acusaron a las autoridades mexicanas participantes de ser traido- 
NOTAS

res, asegurando que asistían a las autoridades estadounidenses en sus intentos de violar la soberanía y la integridad territorial de México. El problema, claro está, no tenía nada que ver con la soberanía ni la integridad territorial, sino con un flagrante intento de un congresista mexicano por obtener capital político.

Esto contrasta de manera muy marcada con la relación generada tras el once de septiembre entre Canadá y Estados Unidos, en particular con la llegada del nuevo gobierno conservador. El gobierno de Stephen Harper ha tomado la tarea de demostrar tanto a quienes están a cargo de las políticas estadounidenses como a la opinión pública que Canadá es de hecho un buen aliado cuando se trata de luchar contra el terrorismo.

Primero y principalmente, la administración de Harper ha extendido la presencia militar canadiense en Afganistán hasta por lo menos el 2009, revocando la promesa de los gobiernos liberales previos de regresar las tropas a casa al inicio de 2007. Actualmente Canadá tiene 2200 tropas en Afganistán, involucradas principalmente en misiones de combate junto con tropas de Estados Unidos y de la OTAN.

Haciendo referencia específica a América del Norte, y con las recientes pruebas nucleares de Corea del
Norte, el gobierno de Harper ha reabierto el debate nacional en torno a si Canadá debe o no participar en el programa de defensa balística de EU; una vez más, algo que el gobierno liberal había descartado por no ser parte del interés nacional. Si el gobierno de Harper decide que Canadá participará en la defensa balística -y cada vez es más claro que lo hará-entonces esto requerirá de una renegociación del tratado bilateral Canadá-Estados Unidos para la Defensa Aérea de América del Norte (NORAD por sus siglas en inglés) el cual fue establecido en 1958 por ambos países.

El NORAD monitorea y rastrea objetos de construcción humana en el espacio; valida y previene ataques contra Norteamérica por parte de aviones, misiles o naves del espacio. El NORAD también provee de vigilancia y control del espacio aéreo a Canadá y Estados Unidos. El comandante en jefe de NORAD es elegido y responde al primer ministro canadiense y al presidente de los Estados Unidos. La sede de NORAD está en la Base de Fuerza Aérea Peterson, en Colorado Springs, Colorado.

Los ataques terroristas del once de septiembre produjeron un cambio en la organización estructural de NORAD, ya que la alianza para la defensa aérea fue incorporada al Northern Command estadounidense 
(NORTHCOM). La misión de NORTHCOM es realizar operaciones para disuadir, prevenir y derrotar amenazas y agresiones dirigidas contra los Estados Unidos, sus territorios e intereses dentro de su área de responsabilidad. Esta área incluye acercamientos aéreos, terrestres y marítimos, así como el territorio continental de Estados Unidos, Alaska, Canadá, México y el agua circundante hasta 500 millas náuticas, además del Golfo de México y los estrechos de Florida. El papel de Canadá dentro de NORTHCOM consiste en asistir en la defensa del espacio aéreo norteamericano, estipulado en el tratado NORAD. Es importante mencionar que NORTHCOM no es considerado como una amenaza para la soberanía canadiense por los tomadores de decisión. Sin duda, porque NORAD es ahora parte de NORTHCOM, la mayoría de los observadores concuerdan en que cualquier renegociación del tratado resultará en una cooperación miltar más cercana entre los dos países, no sólo con respecto a la cuestión aérea, sino también en la defensa costera y territorial. Por ello, la página de internet del Departamento de Relaciones Exteriores afirma que: "El gobierno canadiense continuará desarrollando la cooperación entre Canadá y Estados Unidos en otras áreas, involucrando otros departamentos, incluyendo la coopera- ción en seguridad marítima [...] y con respecto a cuestiones fronterizas".

Canadá también ha alterado a su vez su estructura militar. El primer y más importante cambio fue la noticia de la creación del Comando Canadiense (Canada COM) en febrero de 2006. Canada COM fue creado para atender las realidades de un nuevo ambiente de seguridad nacional que Canadá enfrentaba y para dar mayor énfasis a la defensa de Canadá y de Norteamérica. Igualmente, es la piedra angular del nivel de comando y control de las Fuerzas de Transformación Canadienses. El Comandante de Canada COM le reporta directamente al Jefe del Estado Mayor y es responsable de la conducción de todas las operaciones domésticas, ya sean de rutina o de contingencia, y será la autoridad nacional operativa para la defensa de Canadá y América del Norte. La creación de Canada COM significa que, por primera vez, una cadena de comando unificada e integrada a nivel nacional y regional tendrá autoridad inmediata para desplegar recursos marítimos, terrestres y aéreos en las diferentes zonas regionales de responsabilidad, apoyando operaciones domésticas.

Canadá y los Estados Unidos también han implementado el Inte- 
NOTAS

grated Border Enforcement Team Program (IBETS por sus siglas en inglés) a lo largo de 23 puntos de la frontera. Este programa binacional permite a cinco agencias de seguridad compartir información y trabajar de manera conjunta, diariamente, con otras agencias de reforzamiento, ya sean locales, estatales o provinciales. Estas agencias pueden trabajar en cuestiones relacionadas con seguridad nacional, crimen organizado y crimen que transita por la frontera entre Canadá y Estados Unidos entre los Puertos de Entrada (POE por sus siglas en inglés). Las agencias socias IBETS de Canadá y Estados Unidos son: la Policía Montada (RCMP), the Canadian Border Service Agency (CBSA por sus siglas en inglés), US Customs and Border Protection, the US Bureau of Immigration and Customs Enforcement y the US Coast Guard.

Canadá ha colocado cuatro Equipos para el Reforzamiento de la Seguridad Nacional Integrada (INSETS por sus siglas en inglés) en los centros urbanos principales: Toronto, Montreal, Vancouver y Ottawa. INSETS incrementa la capacidad para recabar, compartir y analizar inteligencia entre las distintas agencias de seguridad canadienses sobre blancos que representan una amenaza a la seguridad nacional. Los INSETS están formados por agentes de la Policía Montada, el Canada
Border Services Agency (CBSA por sus siglas en inglés), Citizenship \& Immigration Canada (CIC por sus siglas en inglés), el Canadian Security Intelligence Service (CSIS por sus siglas en inglés) y servicios policiales en provincias y municipios.

Con respecto a la legislación, Canadá introdujo la ley C-11 que intenta limitar el acceso a las apelaciones por parte de refugiados rechazados $\mathrm{y}$, al mismo tiempo, facilita deportar individuos cuyas peticiones específicas por asilo han sido rechazadas. Con respecto a las actividades terroristas específicamente, el gobierno liberal antecesor introdujo la ley C-36 que dota de recursos adicionales y de poder a las policías para identificar y castigar terroristas y grupos terroristas. Más detalladamente, la ley volvió ilegal el recaudar fondos en nombre de los terroristas; permite al gobierno confiscar bienes de las organizaciones terroristas, y expande la autoridad de la policía para perseguir a terroristas sospechosos.

En suma, el objetivo de esta discusión es demostrar las diferencias tan marcadas que existen entre el acercamiento de Canadá y México en torno a la seguridad de Norteamérica y argumenta que México enfrenta una batalla cuesta arriba en el esfuerzo de ser un buen aliado en la guerra contra el terrorismo. Como se señaló anteriormente, aunque existe el deseo de 
cooperar a nivel federal, ha habido una respuesta operativa mínima. Alternativamente, cuando ha habido una respuesta, ésta ha sido incoherente y manchada por la corrupción. La experiencia del último sexenio puede servir como una lección importante para el actual gobierno de
Felipe Calderón: si la administración mexicana no sigue una estrategia mucho más activa, lo más probable es que México quede fuera de la región en términos políticos, sociales y económicos, dado que Canadá y los Estados Unidos han progresado mucho en los últimos años. 
CITAM Derechos Reservados.

La reproducción total o parcial de este artículo se podrá hacer si el ITAM otorga la autorización previamente por escrito. 